\title{
Metodología para la implementación de un blog corporativo externo
}

\author{
Por Marcos Ros-Martín
}

\begin{abstract}
Resumen: Se propone una metodología para un proyecto de implantación de un blog corporativo externo. En primer lugar, se hace una introducción a los nuevos modelos comunicativos de las organizaciones respecto a sus clientes y usuarios, derivados de la penetración de las nuevas tecnologías en la sociedad y el desarrollo de la Web 2.0. En segundo lugar, se profundiza en el concepto de blog y las ventajas que pueden suponer este tipo de herramientas dentro del ámbito corporativo, además de establecer sus tipos y funciones, para finalmente realizar una propuesta metodológica para que el proyecto de un blog corporativo externo sea exitoso.

Palabras clave: Blogosfera, Blogs corporativos externos, Blogs relacionales, Comunicación empresarial, Empresa 2.0, Weblogs.

\section{Title: Methodology for the implementation of an external corporate blog}

Abstract: A methodology to implement a corporate external blog is proposed. Firstly, there is an introduction to the new models of organizational communication with customers and users, resulting from the acceptance of new technologies in society and the development of Web 2.0. Secondly, it delves into the blog concept and the benefits that this type of tools can afford within the corporate realm, along with their types and functions, to finally propose a methodology for successful corporate implementation of an external blog.

Keywords: Blogosphere, External corporate blogs, Relational blogs, Corporate communications, Business 2.0, Weblogs.

Ros-Martín, Marcos. "Metodología para la implementación de un blog corporativo externo". En: El profesional de la información, 2008, v. 17, n. 5, septiembre-octubre, pp. 502-509.

DOI: $10.3145 /$ epi.2008.sep.03

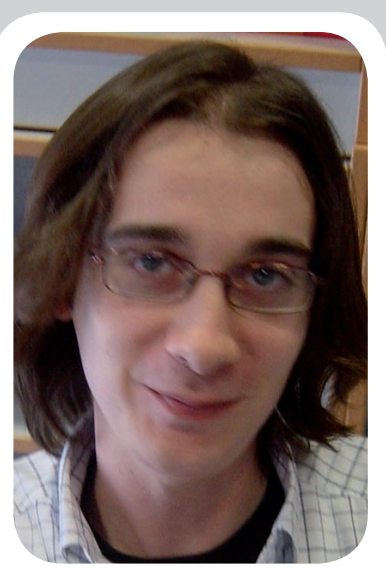

Marcos Ros-Martín, licenciado en documentación por la Universitat Politècnica de València, trabaja como documentalista en la Unidad Técnica de Información de Aidico (Instituto Tecnológico de la Construcción) de Valencia. Previamente fue documentalista informativo en el diario regional "Levante-El mercantil valenciano". Es coeditor del blog personal "El documentalista enredado".
\end{abstract}

\section{Comunicación corporativa en la sociedad post-industrial}

Con la publicación de The Cluetrain Manifesto (Locke, 1999), sus autores se adelantaban a su tiempo ofreciendo una visión de lo que se avecinaba en el ámbito de la comunicación empresarial, tras el progresivo desarrollo de una sociedad en la que cada vez se realiza un mayor número de actividades dentro de un entorno tecnológico.

Concretamente, en el documento "El mercado son conversaciones" se exponen 95 claves para comprender y facilitar la gestión de los nuevos vínculos generados por las nuevas tecnologías entre las empresas, sus clientes y proveedores.

A pesar de que en los años posteriores a su publicación la evolución del comercio electrónico se vería ralentizada tras el estallido de la denominada "Burbuja tecnológica", comenzó a asentarse de forma paralela un nuevo modelo comunicativo en internet ya preconi- zado en el Manifiesto, que se sostendría sobre un nuevo tipo de web -enmarcada en lo conocido posteriormente como "Blogosfera" - y que supondría toda una revolución a la hora de difundir opiniones y compartir información y conocimientos. Tanto es así que la popularización de los blogs como sistemas de publicación en internet de forma abierta y participativa establecería las bases sobre las que se cimentaría la denominada $\mathrm{Web}$ $2.0^{3}$ o colaborativa.

Sin embargo, la progresiva penetración de internet en la sociedad trajo consigo un gran aumento de la información disponible y, por lo tanto, de la cantidad de datos que los internautas debían aprender a manejar. Dentro del concepto la economía de la atención (Gimeno, 2004) -ligado también a la sobrecarga informativa o infoxicación- se profundiza en la idea de que las organizaciones deben tratar de captar el interés del usuario y superar los déficits de atención que el bombardeo continuo de datos a través de distintos soportes provoca en las personas. 
Hasta ese momento las empresas se habían encontrado en un entorno cómodo siguiendo un modelo de comunicación lineal o unidireccional, donde los principales conductores del mensaje corporativo eran los medios de comunicación social mediante notas, ruedas de prensa y publicidad. Empero, este modelo de difusión de la información no aportaba un retorno conveniente sobre el alcance y el impacto de los mensajes que las organizaciones enviaban a su mercado. Además, y por otro lado, nos hallamos dentro de un marco donde los hábitos de los clientes y consumidores se encuentran en perpetua evolución, por lo que ese modelo lineal se torna obsoleto puesto que su impacto real no es evaluable.

Frente a esto comienza a surgir un nuevo modelo comunicativo mucho más flexible y abierto, que fomenta la participación de todos los agentes implicados en él con flujos de información descendentes, desde los departamentos de toma de decisiones a los clientes, y ascendentes, de los clientes a los departamentos y la dirección de las organizaciones. Cornella (2000) propuso unas líneas maestras para atraer y mantener la atención de los usuarios y clientes en una sociedad post-industrial y con una penetración tecnológica avanzada, que se resumían fundamentalmente en tres ideas:

\section{"Los blogs internos mejoran la gestión de la información y el conocimiento, mientras que los externos favorecen las relaciones y las ventas"}

\section{- Atracción}

Hay que disponer de una marca bien posicionada para captar a nuestro público objetivo.

- Búsqueda

Debemos poder ser encontrados fácilmente, tanto nosotros como organización, como nuestros productos y servicios. Obviamente estar posicionados correctamente dentro de los buscadores de internet en general, y en Google en particular, es algo vital para conseguir este propósito en este tipo de sociedad.

- Tropiezo

Tenemos que conocer los hábitos de nuestros clientes para provocar que un encuentro casual sea gratificante para ellos.

Se comienza a considerar a los blogs corporativos como un canal de comunicación alternativo (entre la dirección de la organización, sus empleados, los clien-
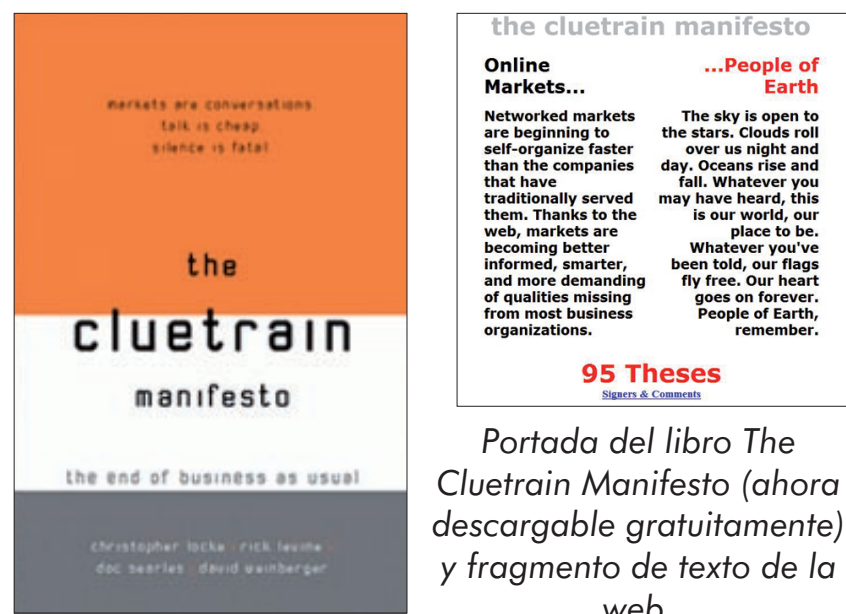

Portada del libro The Cluetrain Manifesto (ahora descargable gratuitamente) y fragmento de texto de la web.

Los autores dieron a su mensaje cierto aire mesiánico algo molesto, pero tuvieron la gran visión de anunciar como sería la Web 2.0, especialmente en lo relativo a los negocios. También hay que decir que hacía ya casi 3 años que existían los blogs. http://www.cluetrain.com

tes existentes y potenciales) que no necesita el filtro de terceras personas (gabinetes de prensa) o de los propios medios de comunicación (Celaya; Herrera, 2006). De este modo el blog se erigiría como una herramienta viable en la consecución del establecimiento de una "conversación" con el público objetivo de una organización, además de atraer la atención de éste.

\section{Blogs corporativos. Ventajas para las organizaciones}

Un blog o weblog podría ser definido como un sitio web "creado por uno o varios autores, generalmente mediante una herramienta sistematizada de gestión de contenidos (content management system o CMS), actualizado con gran frecuencia, presentado habitualmente en tono informal y orden cronológico inverso, con abundancia de hipervínculos a otros blogs y páginas, persistencia de URLs o vínculos para cada entrada realizada (permalinks), y la posibilidad de introducir comentarios por parte de los visitantes, moderados o no por el/los propietarios del blog" (Dans, 2005). Por otra parte y de forma concreta, la definición del blog corporativo consistiría en un "blog publicado por [o con el apoyo de] una organización para alcanzar los objetivos de ésta" (Wacka, 2006).

El hecho de que un weblog sea utilizado dentro de una organización no debe llevarnos a la sorpresa, puesto que se demuestran como unas herramientas de difusión de la información con grandes ratios de éxito si sus políticas de publicación e interacción son convenientemente establecidas y conducidas.

Las ventajas de la utilización de blogs en ámbitos corporativos o institucionales se resumen en (Villanueva, 2007): 


\section{- Bajo coste}

La gran popularidad de este formato en la Web ha fomentado la proliferación de distintos CMS. Sin grandes esfuerzos podemos encontrar sistemas de gestión de pago o gratuitos dependiendo de nuestras necesidades y posibilidades, pero en cualquier caso podemos obtener el software para la gestión de un blog a un precio muy ajustado. Por otro lado, la consolidación del formato y de sus comunidades de desarrollo facilita la obtención de soporte técnico, así como la subsanación de posibles agujeros de seguridad que el sistema pudiese presentar, además de la obtención de nuevas versiones a un precio casi residual.

\section{- Instantáneos y no invasivos}

La publicación en los blogs se realiza de forma completamente instantánea y transparente para el usuario, no es necesario disponer de grandes conocimientos en la edición de páginas web; mientras que la sindicación de contenidos (RSS $)^{4}$ permite que sólo reciban las actualizaciones de forma automática aquellos interesados que se han suscrito previamente.

- Visibilidad y optimización en los buscadores

Los blogs se han venido desarrollando desde un punto de vista $S E O$ (search engine optimization) $)^{5}$, considerando la mejor forma de publicar los contenidos para posicionarse en los buscadores. Por otro lado, y gracias a su filosofía de enlazar a las fuentes, junto a la de actualización constante, son los propios buscadores los que consideran a este tipo de webs como fuentes muy ricas para la obtención y valoración de recursos informativos.

- Proximidad personal

La comunicación y las formas que se utilizan en la blogosfera distan mucho del lenguaje estricto y formal que se emplea en las notas de prensa, aportando un rostro más humano a la organización y fomentando la "conversación" entre los internautas que lo visitan y los editores que lo mantienen.

- Feedback

Fruto de esa "conversación", los comentarios recibidos sirven a la propia organización para la obtención de información externa que puede resultar muy interesante para la elaboración de nuevos productos y/o servicios, además del refuerzo y mejora de los existentes.

\section{- Aprendizaje}

El mantenimiento de un weblog obliga a los editores a mantenerse constantemente actualizados, siguiendo la evolución de la competencia y de sus nuevos productos. También puede fomentarse su utilización en organismos destinados a la $\mathrm{I}+\mathrm{D}$, puesto que fortalecen las actividades paralelas pero necesarias como la vigilancia tecnológica ${ }^{6}$.

\section{- Fidelización}

El establecimiento de este tipo de herramientas ofrece un poderoso medio para la creación de comunidades en torno a un servicio, área temática del conocimiento, producto, o llegar a nichos de audiencia no cubiertos por otros medios. Al mismo tiempo se puede dar soporte a los usuarios y clientes de la organización.

\section{- Networking}

Los weblogs incrementan las relaciones, ampliando las ya existentes con profesionales interesados en el trabajo que se está llevando a cabo dentro de la organización.

\section{- Autoridad}

La publicación de informaciones relativas a un campo determinado de investigación o del conocimiento aumenta la autoridad del equipo dentro del mismo, posicionando a la organización como experta.

\section{"La blogosfera considera lícita la creación de un blog para aumentar las ventas siempre que sea transparente y no se distorsione su finalidad"}

\section{Tipos de blogs y sus objetivos}

De forma previa al detalle de los usos de los blogs corporativos, estableceremos su clasificación. Por supuesto que existen múltiples formas de segmentarlos, pero de una manera sencilla pueden ser clasificadas fundamentalmente en tres tipos (Orihuela, 2006):

- Personales

Reflejan los pensamientos y opiniones de un individuo. Se trata de un diario en su forma digital donde se detallan las reflexiones, opiniones y recomendaciones de una persona. Su público objetivo principal se circunscribe al círculo más íntimo del autor.

\section{- Temáticos/profesionales}

Se especializan en una disciplina o una temática y los textos son escritos a título personal por un profesional. Dentro de ellos podemos encontrar a líderes de opinión de cada nicho. Encuadramos en esta categoría los blogs comerciales que se dedican a la explotación de las nanoaudiencias?

- Corporativos

Publicados y mantenidos por organizaciones para la consecución de su misión y objetivos. 


\section{Tipos de blogs corporativos}

Pueden ser categorizados según el público al que se dirigen. Así, nos encontraríamos con dos tipos principales: internos y externos.

Los internos, sin querer profundizar en sus usos, tienen dos finalidades principales: la mejora de la comunicación interna y la colaboración entre los equipos de la organización; y facilitar la gestión de la información y el conocimiento.

Los externos tendrían el propósito de mejorar las ventas y el reforzamiento de las relaciones entre la organización y el entorno en el cual desarrolla sus actividades.

Este último tipo se puede clasificar atendiendo a una serie de criterios (Villanueva, 2007):

- De marca

Para introducir una marca nueva, cambiarla o reforzarla. Se trata de una de las modalidades más extendidas dentro de los blogs de empresa. Sería el caso de los de General Electric, South West Airlines o el español Weblogs $S L$ que sirve como refuerzo a la imagen de los distintos blogs comerciales que mantiene esta empresa.

\section{http://www.grcblog.com/ \\ http://www.blogsouthwest.com/ \\ http://www.weblogssl.com/ \\ - De producto o servicio}

Dan soporte al lanzamiento de un nuevo producto, seguimiento durante su vida comercial, anuncio de mejoras y novedades, y la obtención de feedback. Por ejemplo, el conjunto de blogs que mantiene Google de sus productos (como los dedicados a Gmail o AdSense), o el de Security Response de Symantec.

- De trabajadores

Mantenidos tanto por directivos como por empleados dentro del dominio web corporativo o en dominios externos, pero siempre reconocidos por la empresa como tales. Por ejemplo, el blog de Jesús Encinar, CEO del portal inmobiliario Idealista; el de Julio Alonso, de Weblogs $S L$; o el de John Mackey, de Whole Foods Market.

http://gmailblog.blogspot.com/

http://adsense-es.blogspot.com/

http://www.symantec.com/business/security_ response/weblog/index.jsp

http://www.jesusencinar.com/

http://www.merodeando.com/

http://wholefoodsmarket.com/socialmedia/jmackey
- De eventos

Promoción de actividades durante la preparación, lanzamiento y ejecución de los mismos. Ejemplos: blog de las 11es. Jornades Catalanes d'Informació i Documentació organizadas por el Col-legi Oficial de Bibliotecaris-Documentalistes de Catalunya, o el del Workshop Calsi 2007, de la Univ. Politécnica de Valencia.

http://www.cobdc.org/jornades/11JCD/bloc/

http://www.calsi.org/2007/

- Sectoriales

Seguimiento de las evoluciones del sector donde se despliega la actividad económica y profesional, reforzando de este modo la imagen como expertos. Por ejemplo, eTc El blog de marketing en español que mantiene la empresa Territorio Creativo, o el de Sedic (Asociación Española de Documentación e Información).

http://etc.territoriocreativo.es/

http://blog.sedic.es/

\section{Objetivos de los blogs corporativos externos}

Sin ser excluyentes los unos de los otros, podrían ser:

- Comunicación

Difusión de actividades que realiza la organización, participación en proyectos y/o investigaciones, etc.

- Posicionamiento de la organización como experta en un mercado

Esto se realizaría tomando el pulso a la actualidad de un mercado. Por ejemplo, comentando las recientes publicaciones científicas y técnicas, los nuevos productos y servicios de la competencia, noticias de prensa, etc.

- Reclutamiento de personal

Uno de los objetivos a la hora de posicionarnos también pasa por tratar de atraer a los mejores talentos disponibles en el mercado laboral, mostrando ser una organización activa y dando a conocer los trabajos realizados dentro de la organización o de un departamento de la misma.

- Establecimiento de relaciones

Anunciando la presencia en un acto, congreso o jornada; se establece la posibilidad de contactar con los

"La aparición de la figura 'chief blog officer' (CBO) puede ser indicativa de que el formato blog se está asentado en la comunicación corporativa" 
miembros a través de redes sociales o el fortalecimiento de contactos ya realizados.

\section{Propuesta metodológica}

La blogosfera dispone de cierto savoir faire con respecto al comportamiento y maneras dentro de ella. Así, por ejemplo, una organización nunca debería crear un $f l o g$ (fake blog $)^{8}$ para la promoción de sus productos, si no quiere ser denostada y provocar a corto plazo una crisis de comunicación. Una de las principales características de la blogosfera es la transparencia y, por ello, se considera que la creación de un blog para fomentar las ventas es lícito siempre que no se distorsione y se manipule la realidad; es decir, se debe especificar claramente la finalidad de ese sitio web. Esa hostilidad que, aparentemente, desprende la blogosfera ante las organizaciones que se sirven de ella para la obtención de beneficios sin tener presente su idiosincrasia, es una de las principales barreras que se perciben por parte de las organizaciones a la hora de utilizar su potencial.

Por supuesto que existen instituciones que usan los blogs de una forma exitosa. De hecho, en un primer momento fueron las empresas tecnológicas -por ejemplo, Sun- las que adoptaron el modelo blog como elemento comunicativo, aunque en un principio se trató de iniciativas individuales y no coordinadas.

http://www.sun.com/aboutsun/media/blogs/index. jsp

Sin embargo, hay que considerar que la actividad blogger de una organización debe de ser convenientemente conducida y las líneas maestras de sus objetivos trazadas. De lo contrario, el proyecto de instauración de un blog, o de un conjunto de ellos si fuese el caso, podría estar condenado al fracaso.

La reciente aparición de la figura del chief blog -O blogger-officer (CBO) (Financial week, 2008) en Estados Unidos puede ser un indicador de que el formato blog se está asentado como modelo comunicativo y sus formas normalizadas. Por otro lado, en España, la implantación de blogs corporativos comienza a ser divulgada en la prensa generalista (Verdeja, 2007), así como la percepción de que es necesaria la figura de un experto para la coordinación y supervisión de los contenidos.

Igualmente, hay que tener presente que si es una institución dedicada a la investigación (una universidad o un centro tecnológico) la que se decide a desarrollar este modelo de comunicación, puede encontrarse con distintas finalidades y necesidades atendiendo a los distintos departamentos o secciones que la componen ${ }^{9}$, por lo que la maduración de una política común en la edición de sus contenidos es completamente necesaria para seguir una línea de publicación similar en todos los casos.
Así pues, se propone una metodología para la implantación de un blog corporativo externo siguiendo los distintos estudios realizados hasta la fecha (Edelman, 2007; Cass, 2008; Olson, 2007), además de los consejos publicados (Baker, 2005; Celaya, 2007, Holtz, 2006; Ortiz, 2008; Scoble, 2003; Villanueva, 2007), y la experiencia propia.

\subsection{Establecimiento de objetivos}

El surgimiento de la iniciativa de comenzar a publicar un blog no tiene por qué ser arriba a abajo, es decir, de la dirección a sus departamentos, sino más bien es probable que suceda al contrario, que un departamento lo proponga a la dirección y se extienda posteriormente al resto de la organización. Obviamente, la apertura de un nuevo modelo comunicativo puede provocar reticencias que deben ser correctamente conducidas, por lo que el apoyo de la dirección es vital para el éxito de una actividad de estas características. Cuando el proyecto entre en consideración se deberá establecer una serie de propuestas y alternativas.

Lo primero que se debe definir es la finalidad, qué se espera de él (aunque posteriormente evolucione hacia otros objetivos), así como el establecimiento de una política global de comunicación dentro de la Red. Por ejemplo, puede suceder que desde la dirección no se contemple la utilización de un subdominio en la web corporativa, por lo que habrá que considerar la adquisición de un dominio propio tanto si es el caso del blog como un grupo de blogs ${ }^{10}$.

Hay que señalar que no es recomendable la utilización de lugares de registro gratuito (Wordpress.com, LaCoctelera.com o Blogger.com) para la realización de este tipo de proyectos. Por ello, habrá que llevar a cabo un análisis de los $C M S$ disponibles en el mercado para seleccionar el más adecuado atendiendo a las problemáticas y a los elementos que queramos incorporar, junto a las características técnicas de los servidores web de los que dispongamos, además de los futuros proyectos que deseemos implementar.

\subsection{Estudio de buenas prácticas}

Seguramente, a la hora de plantearse un blog, la dirección de una organización establecerá dos barreras previas: el ROI (return of investment) ${ }^{11} \mathrm{y}$ las implicaciones legales derivadas de su apertura.

El software de gestión de un blog tiene un coste casi nulo, pero no así su mantenimiento y su personalización. El diseño de una plantilla específica puede tener un coste de unos miles de euros, pero se trata de un gasto que resulta necesario asumir para disponer de cierta entidad y personalidad propias dentro de la blogosfera. También habrá que tener presente que los empleados que se designen para mantener los contenidos dedicarán una parte de su horario laboral a esta tarea, por lo 


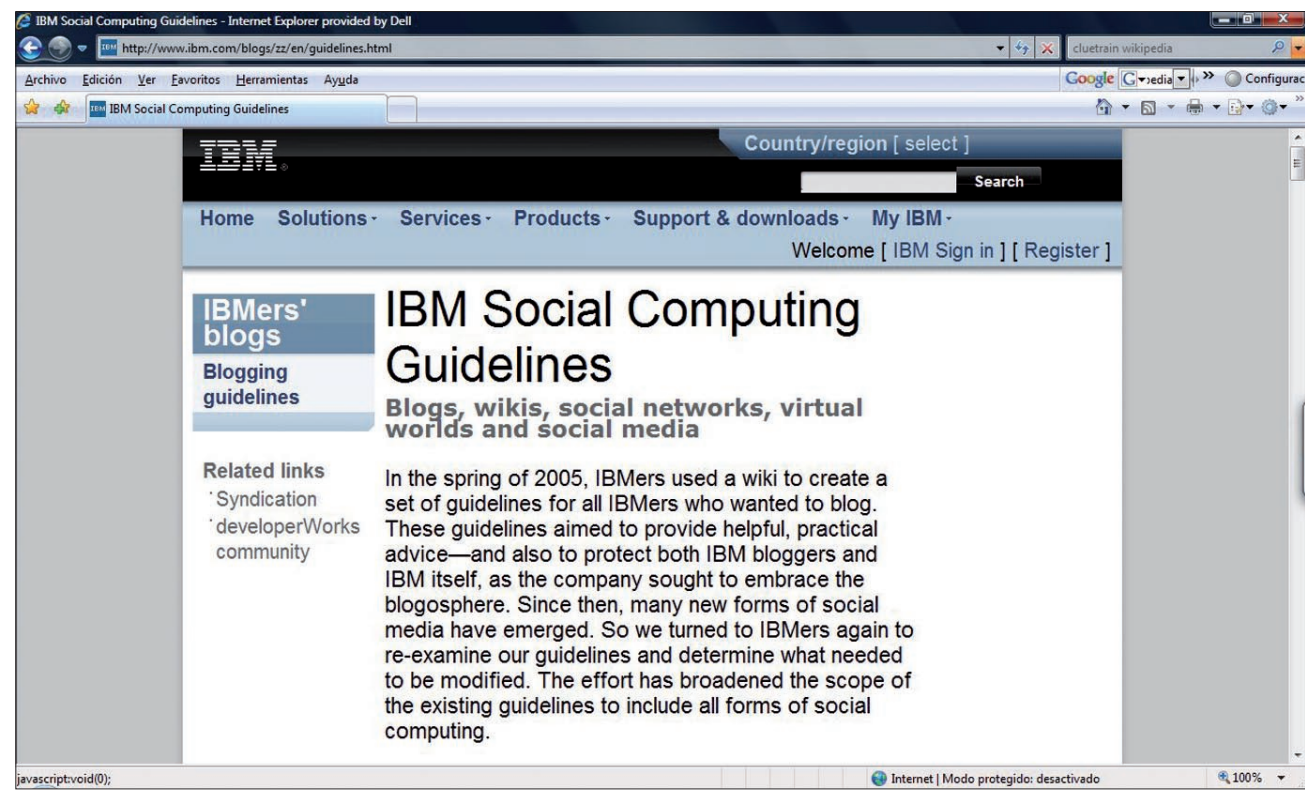

que habrá que tener presente su coste. Finalmente, será conveniente la realización de un pequeño estudio para comprobar qué está haciendo nuestra competencia, si es que se desconoce, con el objetivo de detectar aquellas temáticas que despiertan más interés dentro de la blogosfera.

Sobre las implicaciones legales, dependiendo del tipo de organización, se deberán examinar distintas aspectos. Por ejemplo, si el blog es para un departamento de investigación y desarrollo, habrá que tener presente que todo lo que se publique en él puede derivar en problemas a la hora de presentar una patente al no cumplir el precepto de novedad del invento por haberse divulgado ya en el blog. Si se trata de un organismo público deberemos estudiar que tal vez desde fuera de la institución el blog se considere como vehículo oficial de comunicación de la misma, algo que no debiese suceder en ningún caso, diferenciando los niveles de oficialidad y modos comunicativos.

Asimismo, se debe tener en cuenta que los comentarios que se publiquen pueden provocar que la organización se encuentre con problemas legales si no se especifican distintas cláusulas de aviso o notas legales tanto para los editores como para los usuarios que aporten contenido. Esto es importante tanto en el caso de blogs profesionales como corporativos y es una práctica ampliamente extendida dentro de la blogosfera.

\subsection{Manual de estilo}

Hay que considerar que las personas que van a publicar los contenidos probablemente sean investigadores y profesionales expertos en las materias de la empresa, no periodistas ni comunicadores. Aunque algunos tuviesen experiencia en la blogosfera o en medios de comunicación sociales o científicos, es recomendable la redacción de un manual de estilo para fijar el enfoque que se quiere dar a la web. Habrá que detallar qué se quiere hacer con el blog, qué se espera de él, qué se puede (o no) publicar, cómo es la manera más correcta de contestar los comentarios que sean críticos con la organización (si se produjesen), el tono de las informaciones, etc.

Para las actividades blogger en un entorno corporativo podemos guiarnos por la IBM Social Computing Guidelines ${ }^{12}$, en la que se recogen algunos consejos de la multinacional a sus empleados sobre su comportamiento en la Web 2.0.

\section{"Un blog deber ser fresco e inmediato, por lo que su edición no debe ser coartada por la supervisión permanente de un cargo superior a los editores"}

\subsection{Coaching $^{13}$ para los redactores}

Los redactores deben considerar el blog corporativo como algo propio y una ventana al exterior hacia sus compañeros de profesión y clientes. Teniendo en cuenta que la publicación puede provocar en un principio algunas incomodidades a los editores por la responsabilidad que se pueda percibir que representa, sería conveniente animar a participar a aquellos que fuesen más proactivos como evangelizadores dentro de la organización o del departamento.

Por otro lado, se potenciará que cada cual firme las informaciones como propias, ofreciendo al mismo tiempo la alternativa de publicar de una forma genérica las comunicaciones más corporativas. Se puede guiar a los redactores sobre qué tipo de contenidos pueden o deberían publicar, dependiendo del ámbito sobre el que trabajen. Las informaciones que se podrían divulgar pasarían por (Roca, 2008):

\section{- Herramientas y recursos}

Textos que harían referencia a herramientas disponibles que podrían resultar de interés para los lectores o el público objetivo del blog. Por ejemplo, un departa- 
mento dedicado a las TICs podría publicar información sobre nuevo software de programación o aplicaciones para la mejora de la productividad.

\section{- Lecturas y conceptos}

Para reforzar la imagen de expertos en una materia, los editores podrían realizar comentarios de lecturas de artículos científicos, nuevos conceptos surgidos, reseñas de congresos a los que hayan asistido, etc.

\section{- Experiencias y buenas prácticas}

Comentar de forma sucinta experiencias de trabajo reales o buenas prácticas detectadas. Dentro de este campo hay que ser cautos, como se ha dicho, si se quiere patentar algo, o realizar nuevos proyectos de investigación.

\section{- Entrevistas y testimonios}

El contacto con otros profesionales y los comentarios respecto a conversaciones informales pueden servirnos como inicio a un debate e invitar a nuestros lectores a participar en él. Sin embargo hay que tener presente que las conversaciones privadas son delicadas y habrá que consultar con el interlocutor si considera que se está invadiendo la privacidad y la confianza ajenas.

- Actualidad y novedades. Las lecturas de blogs similares pueden permitirnos estar completamente al día y ser los primeros en publicar o comentar sobre nuevos hitos. Esto constituiría un punto a nuestro favor como expertos en una materia.

- Búsqueda de partners. Si se está llevando a cabo un proyecto y se necesita un socio tecnológico o financiero, el blog es una buena forma de encontrar uno.

\subsection{Tutelaje previo}

Todos los comienzos son difíciles y se tienen que romper miedos y esquemas preestablecidos. Para iniciar el proceso de publicación, se puede proponer la práctica con un blog de pruebas off-line donde se corrijan comportamientos y se censure aquello que se perciba como incorrecto. Hay que tener presente que un blog es algo fresco e inmediato, por lo que no debe de ser coartado por la supervisión permanente de un cargo superior a los editores. Si se desea que la comunidad alrededor de la web crezca, es necesario ofrecer cierto margen a los redactores para que innoven por ellos mismos y ensayen nuevas propuestas. Posteriormente se pueden incorporar esos textos de pruebas al blog definitivo.

\subsection{Animador y relaciones externas}

Evidentemente, alguien debe encargarse de lo que en la blogosfera se denomina gardening: cuidar que los comentarios sean pertinentes, que no se extralimiten en lo que se considera correcto, así como la eliminación de comentarios de spammers o trolls ${ }^{14}$.

Aunque se trate de una administración compartida, debe existir un responsable del blog, si bien este perfil todavía se encuentra por definir, a pesar de la existencia del citado cargo de $C B O$. En cualquier caso el administrador de un blog podría ser un profesional de relaciones públicas -periodista o responsable de marketing-, pero que tenga conocimientos de comunicación en internet, o bien una persona con una experiencia previa dentro de la Red y sepa evaluar el funcionamiento del blog convenientemente -un documentalista con experiencia en usabilidad y $S E O-$.

En definitiva, a modo orientativo, se trataría de una persona que conozca el mundo de la blogosfera, que entienda cómo se mueve y cómo se establecen los vínculos dentro de ella, que incite a los editores a que publiquen y ensayen nuevas aproximaciones para que el blog sea exitoso. Igualmente, vigilará la limpieza del blogroll $^{15}$, si existiese, y establecería las relaciones externas con otros blogs similares, si se considerase pertinente hacerlo. Finalmente, deberá ser evangelizador dentro de la organización para que los editores no se desanimen en los primeros momentos, así como monitorizar el funcionamiento del mismo, evaluar los éxitos y fallos, y elaborar informes para la mejora continua.

\section{Notas}

1. Burbuja tecnológica, también denominada Burbuja.com, fue una corriente especulativa que tuvo lugar entre los años 1997 y 2001. Las bolsas de comercio occidentales favorecieron un rápido aumento del valor de las acciones de las empresas vinculadas a internet y a la llamada Nueva economía. A estas empresas, se las conocía como punto-coms puesto que fundamentalmente basaban su modelo de negocio en el comercio electrónico y las expectativas de expansión del mismo. Fuente: Wikipedia. Consultado: 25-6-2008.

http://es.wikipedia.org/wiki/Burbuja_.com

2. Está aceptado que el término weblog o blog fue acuñado en 1997 por Jorn Berger. Por otro lado, el término blogosfera agrupa la totalidad de weblogs y se deriva de la palabra inglesa blogosphere. Debido a que los blogs están conectadas por medio de enlaces, comentarios, históricos y referencias han creado y definido su propia cultura. Por lo tanto, la blogosfera como palabra y concepto es inherente a los weblogs.

Fuente: Wikipedia. Consultado: 27-8-2008.

http://es.wikipedia.org/wiki/Blogosfera

3. Concepto desarrollado por O'Reilly (2005) y el más aceptado actualmente.

4. RSS es un sistema para redifundir noticias y nuevos contenidos a suscriptores de un sitio web, sin necesidad de usar un navegador.

http://es.wikipedia.org/wiki/Rss

5. La tarea de ajustar la información de las páginas que se pretenden hacer aparecer en primeras posiciones de los resultados es conocida como $S E O$ (search engine optimization), optimización para motores de búsqueda. Consiste en aplicar diversas técnicas para lograr que los buscadores de internet sitúen determinada página web en una posición y categoría alta (primeras posiciones) dentro de su página de resultados para determinados términos y frases clave de búsqueda.

Fuente: Wikipedia. Consulta: 26-6-2008.

http://es.wikipedia.org/wiki/Optimizaci\%C3\%B3n_para_Motores_de B\%C3\%BAsqueda 
6. La vigilancia tecnológica es una forma sistemática de captación y análisis de información científico-tecnológica que sirve de apoyo en los procesos de toma de decisiones.

7. Nanoaudiencias es un concepto muy relacionado con los blogs e internet. Consiste en los ámbitos informativos que los medios de comunicación sociales no cubren puesto que el coste económico y el interés por cubrir esos ámbitos no resulta rentable, ya que la audiencia a la que pueda llegar a interesarle es residual.

8. Fake blog o flog es una herramienta de marketing y de relaciones públicas diseñada por profesionales para la promoción de un producto o servicio que podría pasar como un sitio de un entusiasta en forma de blog. El propósito de los flogs es favorecer el marketing viral o la creación de un meme de internet que genere un tráfico y un interés que la empresa no podría obtener por los métodos tradiciones de publicidad.

Basado en Wikipedia. Consulta: 25-6-2008.

http://en.wikipedia.org/wiki/Fake_blog

9. Véanse los blogs de la Universitat Autònoma de Barcelona. http://blogs.uab.cat/

10. Véase los blogs del portal de información universitaria Universia. http://universiablogs.net

11. Retorno de la inversión

12. IBM. Consulta: 26-8-2008

http://www.ibm.com/blogs/zz/en/guidelines.html

13. En el entorno empresarial y personal se conoce por coaching al proceso interactivo y transparente mediante el cual el coach o entrenador y la persona o grupo implicados en dicho proceso buscan el camino más eficaz para alcanzar los objetivos fijados usando sus propios recursos y habilidades. Fuente: Wikipedia. Consulta: 24-7-2008. http://es.wikipedia.org/wiki/Coaching

14. En la jerga de internet, un troll (a veces trol) es un mensaje u otra forma de participación que busca intencionadamente crear controversia y provocar reacciones predecibles, especialmente por parte de usuarios novatos, con fines diversos, desde el simple divertimento hasta interrumpir o desviar los temas de las discusiones, o bien provocar flame wars (guerras encendidas) enfadando a sus participantes y enfrentándoles entre sí. Fuente: Wikipedia. http://es.wikipedia.org/wiki/Troll_(Internet)

15. Un blogroll es una colección de enlaces de blogs y otras webs, normalmente presentado dentro de un blog en una columna lateral de la página web.

Basado en: Wikipedia. Consulta: 26-6-2008.

http://es.wikipedia.org/wiki/Blogroll

\section{Bibliografía}

A corporate guide to the global blogosphere. The new model of peer-to-peer communications. Publicado: 11-1-2007. Consultado: 21-6-2008. http://www.edelman.com/image/insights/content/whitepaper011107sm.pdf

Alonso, Julio. "Blogs y empresas". En: Rojas Orduña, Octavio Isaac Blogs. La conversación en internet que está revolucionando medios, empresas y ciudadanos. Madrid: Esic, 2005, pp. 165-218. ISBN: 84-7356-427-8

Baker, Stephen; Green, Heather. "Blogs will change your business". En: Business week, mayo 2005. Consultado: 25-6-2008.

http://www.businessweek.com/magazine/content/05_18/b3931001_mz001. htm

Cass, John; Walter, Carl. "Media blogging success study". En: Northeastern University and backbone media, Publicado: 2-11-2008. Consultado: 20-6-2008.

http://www.scoutblogging.com/success_study/

Celaya, Javier; Herrera, Pau. Comunicación empresarial 2.0. La función de las nuevas tecnologías sociales en la estrategia de la comunicación empresarial. Barcelona: Grupo BPMO, 2007. ISBN: 978-84-611-9437-7

Cornella, Alfons. Infonomia!com: La empresa es información. Bilbao Deusto, 2000. ISBN: 84-234-1760-3

Dans, Enrique. "Blogs y empresa: de la oscuridad a la luz, pasando por las tinieblas”. En: Cerezo, José Manuel. La blogosfera hispana. Madrid: Fundación Orange, 2006, pp. 158-169. Consultado: 24-06-2008. http://www.fundacionorange.es/imagenes/areas/25/253/blogosfera.jpg

Dans, Enrique. "Blogs y empresa. Una aproximación a la vanguardia de la blogosfera corporativa". En: Telos: Cuadernos de comunicación, tecnología $y$ sociedad, n. 65, 2005, pp. 78-85. Consultado: 24-6-2008.

http://www.campusred.net/telos/articulocuaderno.asp? idarticulo $=8 \& \mathrm{rev}=65$

Gimeno, José-Antonio. "La economía de la atención”. En: La Flecha. Tu diario de ciencia y tecnología, publicado: 2-8-2004. Consultado: 25-6-2008. http://www.laflecha.net/articulos/blackhats/economia_atencion/

Hiltz, Shel; Demopoulos, Ted. Blogging for business. Everything you need to know and why you should care. Chicago: Kaplan, 2006, pp. 113-119. ISBN: 978-1-4195-3645-8

Locke, Christopher; Levine, Rick; Searls, Doc; Weinberger, David. The Cluetrain Manifesto: The end of business as usual. Consultado: 21-6-2008. http://www.cluetrain.com/book/index.html

Olson, Dave; Janzen, Gordon. "Blogging for retailers, White paper - Why it matters and how to get started". En: Get elastic. The ecommerce blog, publicado: 23-1-2007. Consultado: 20-6-2008.

http://www.getelastic.com/blogging-retailer-white-paper/

O'Reilly, Tim. What is Web 2.0 - Design patterns and business models for the next generation of software. En: O'Reilly, publicado: 30-09-2005. Consultado: 21-6-2008.

http://www.oreillynet.com/pub/a/oreilly/tim/news/2005/09/30/what-is-web20.html

Orihuela, José Luis. La revolución de los blogs. Madrid: La esfera de los libros, 2006. ISBN: 8497344987

Ortiz de Zárate Tercero, Alberto. Manual de uso del blog en la empresa. Cómo prosperar en la sociedad de la conversación. Barcelona: Zero Factory, 2008. ISBN: 978-84-612-1535-5. Consultado: 22-6-2008. http://www.infonomia.com/img/libros/pdf/BlogsEmpresa.pdf

Roca, Genís. "Consejos prácticos para blogs profesionales y de empresa". En: Genís Roca, Publicado: 22-3-2008. Consultado: 21-6-2008. http://www.genisroca.com/2008/03/22/consejos-practicos-para-blogsprofesionales-y-de-empresa/

Rojas-Orduña, Octavio-Isaac. "Blogs y relaciones públicas". En: Rojas-Orduña, Octavio-Isaac. Blogs. La conversación en internet que está revolucionando medios, empresas y ciudadanos. Madrid: Esic, 2005. pp. 219-281. ISBN: 84-7356-427-8

Scoble, Robert. "The corporate weblog manifesto". En: Scobleizer. Microsoft Geek Blogger, publicado: 26-2-2003. Consultado: 20-6-2008. http://scoble.weblogs.com/2003/02/26.html

Verdeja, Álvaro. "Comunicación muy personal”. En: El pais, publicado: 28-1-2007. Consulta: 20-6-2008.

http://www.elpais.com/articulo/semana/Comunicacion/personal/ elpepueconeg/20070128elpneglse_4/Tes

Villanueva, Julián; Aced, Cristina; Armelini, Guillermo. "Los blogs corporativos: una opción no una obligación". En: Cuadernos del EBCenter. Consulta: 23-06-2008.

http://www.divshare.com/download/1420234-e2f

"Virtual reality: Chief blogger title catching on with corporations, Companies keen to engage consumers in the blogosphere; 'lightning rods'?". En: Financial week, publicado: 29-4-2008. Consultado: 25-6-2008.

http://financialweek.com/apps/pbcs.dll/article? AID =/20080429/ REG/740115194/1015/investorrelations

Wacka, Fredik. "The beginners' guide to corporate blogging". En: Your guide to corporate blogging, publicado: 1-11-2004. Consultado: 22-6-2008. http://www.corporateblogging.info/2004/11/free-corporate-bloggingprimerasp

Marcos Ros-Martín, Instituto Tecnológico de la Construcción (Aidico), Paterna, Valencia.

marcos.ros@aidico.es

marcos@documentalistaenredado.net 
